Check for updates

Cite this: RSC Adv., 2017, 7, 54717

\title{
Oat bran $\beta$-glucan improves glucose homeostasis in mice fed on a high-fat diet $\uparrow$
}

\author{
Yuliang Cheng, (D) Jie Zhang, Kaiyun Luo and Genyi Zhang*
}

Oat-based functional foods are commonly produced with 1,3- $\beta$-D-glucan-enriched oat bran in which $\beta$ glucan (termed as oat bran $\beta$-glucan (OB- $\beta$-glucan)) not only co-exists with multiple other food components but also exists in different physical forms compared to extracted $\beta$-glucan. In the current study, the impacts of OB- $\beta$-glucan on the glucose homeostasis of mice fed on a high-fat diet were investigated to determine whether oat bran provided additional functional metabolic effects compared to extracted $\beta$-glucan. The experimental results using a C57BL/6J mouse model showed that high-fat (HF) diet treatment significantly increased the body weight (25.1\%), fasting blood glucose (17.4\%), glycated hemoglobin ( $\mathrm{HbA1c}$ ) (31.6\%) and insulin resistance when compared to mice fed on a low fat diet (LF) after treatment for six weeks. In contrast, both OB- $\beta$-glucan and its extracted counterpart with the same amount of $\beta$-glucan (4\%) in the HF-diet significantly improved the insulin sensitivity with reduced levels of $\mathrm{HbA1c}$ and body weight gain. The activation of liver glucokinase (GK) and intestinal phosphoenolpyruvate carboxykinase (PEPCK), and an increased ratio of neuro-peptide proopiomelanocortin (POMC) to neuropeptide $Y$ (NPY) suggest a gut-liver-brain axis mechanism to improved insulin sensitivity and glucose homeostasis after treatment by either OB- $\beta$-glucan or its extracted counterpart. In conclusion, OB- $\beta$-glucan, even in a different physical form, exerted similar physiological effects compared to extracted $\beta$-glucan, and the $\beta$-glucan is the predominant functional component in the health effects of oat bran on glucose homeostasis.

Received 20th September 2017 Accepted 22nd November 2017 DOI: 10.1039/c7ra10437e

rsc.li/rsc-advances molecular weight oat $\beta$-glucan also altered the composition of microbiota to reduce the risk factor of cardiovascular disease. ${ }^{15}$ Thus, there have been extensive studies on the physiological functions of $\beta$-glucan natively presented in oat flour or in its extracted forms. However, considering the multiple components in oat bran in its native physical form, it is necessary to identify whether oat bran provided additional functional metabolic effects compared to extracted $\beta$-glucan.

Oat $\beta$-glucan is a homopolymer of D-glucopyranose units linked through $\beta-(1 \rightarrow 3)$ and $\beta-(1 \rightarrow 4)$ glycosidic linkages arranged in cellotriosyl (DP3) and cellotetraosyl residues (DP4).${ }^{16}$ It is a viscous fiber, and its viscosity, which is determined by its molecular weight, concentration, and solubility, has been widely considered to be the critical rheological property related to its health benefits. Botanically, $\beta$-glucan is predominantly located in the internal aleurone and subaleurone cell walls of the oat grains, and as a natural cell wall component, $\beta$-glucan is tightly associated with the cell wall matrix formed by cellulose, lignin and other polysaccharides such as arabinoxylan. ${ }^{17}$ Once the $\beta$-glucan is isolated, its association with cell wall matrix is completely disappeared. Thus, the extracted $\beta$-glucan and its counterpart presented in the cell wall in cereal grains are different in their physical properties, and reduced solubility of $\beta$-glucan from the cell wall matrix would diminish its physiological functions. However, our
State Key Laboratory of Food Science and Technology, School of Food Science and Technology, Jiangnan University, 1800, Lihu Ave, Wuxi, 214122, Jiangsu Province, PR China. E-mail: genyiz@gmail.com; Tel: +86-0510-85328726

$\dagger$ Electronic supplementary information (ESI) available. See DOI: 10.1039/c7ra10437e 
previous study found that $\beta$-glucan natively presented in the oat grain fraction with a large particle size, which was a type of oat bran based on the AACC standard, significantly decreased the in vitro starch digestibility and postprandial glycemia while the extracted $\beta$-glucan was less effective. ${ }^{\mathbf{1 8 , 1 9}}$ The mechanistic study showed that both protein and $\beta$-glucan played an important role to the in vitro starch digestibility and the rheological properties of the oat flour. Thus, $\beta$-glucan extracted from oat grain and OB$\beta$-glucan (co-existed with many other components) are two different types of $\beta$-glucan. Although the fundamental importance of $\beta$-glucan to the induced health benefits has been widely accepted as discussed above, there are also reports showing no function of $\beta$-glucan in cholesterol metabolism and glucose tolerance. ${ }^{20,21}$ The study of the pasting property of oat flour and its bile acid binding capability also showed the importance of interactions among starch, protein, and $\beta$-glucan. ${ }^{22}$ Additionally, the oat bran is a food processing-related term without exact composition requirement according to the standard, and its processing-dependence implies the difficulty to produce the exact same oat bran samples in both composition and physical form (such as particle size) in the industrial production. ${ }^{18}$ Thus, the possible influence on health benefits by OB- $\beta$-glucan, as compared to extracted $\beta$-glucan, needs to be examined in order to have a better understanding of the health function of $\beta$-glucan natively presented in oat bran.

Obesity and type 2 diabetes have become a public health concern, and insulin resistance related abnormality of glucose homeostasis with elevated blood glucose is a common phenomenon in obese and diabetic patients. ${ }^{23}$ Oat bran is often used to make $\beta$-glucan-enriched food products for improved glycemic control in type 2 diabetic patients, and the extracted $\beta$-glucan has also been shown effective to reduce the postprandial glycemia or the glycemic index of foods. ${ }^{10,24,25}$ However, the different physiochemical properties and physical forms between extracted $\beta$-glucan and the OB- $\beta$-glucan, as discussed above, suggest there might be of differences in their bioactivities. ${ }^{19}$ But there are not many studies specifically focusing on the possible influence of the physical form of $\beta$-glucan on its health benefits. Hence, the aim of the current study was to investigate the impact of $\beta$-glucan in different physical forms on the physiological response through comparing the differences in health efficacy of purified $\beta$-glucan and OB- $\beta$-glucan presented in oat bran.

\section{Materials and methods}

\section{Preparation and characterization of oat bran and extracted $\beta$ - glucan}

Whole oat grains (Hebei, China) were milled into flour, and flour fraction retained after passing through a sieve with a pore size of $250 \mu \mathrm{m}$ (mesh size of 60 ) was used as OB- $\beta$-glucan sample after the endogenous glucanase was deactivated following the method from literature. ${ }^{26}$ The particle size of the sample was measured by a laser particle size distribution instrument (Microtrac S3500, USA). The proximate analysis was carried out based on the standard official method (AACC method 08-01.01 for ash, 30-25.01 for fat, 44-16.01 for moisture, 46-10.01 for protein) from the $11^{\text {th }}$ edition of AACC International. Starch content was measured by a heat-stable $\alpha$-amylase and amyloglucosidase method using the Total Starch Measurement kit (Megazyme International Ireland), and the content of $\beta$-glucan was measured by AACC method 32-22.01 involving lichenase and $\beta$-glucosidase to convert extracted $\beta$-glucan into glucose, and then the glucose content was measured by a GOPOD kit (Sigma, Shanghai China) according to the manufacturer's instructions, and the total glucose content was converted to the content of $\beta$-glucan by factor of 0.9 .

For the characterization of $\beta$-glucan, oat $\beta$-glucan was first extracted based on a literature report, and the purity of extracted $\beta$-glucan was measured by the AACC method 32-22.01 as above. ${ }^{27}$ In a typical procedure, $20 \mathrm{~g}$ oat flour was refluxed with $200 \mathrm{~mL} 82 \%$ ethanol $(\mathrm{v} / \mathrm{v})$ for $2 \mathrm{~h}$ at $85{ }^{\circ} \mathrm{C}$. The mixture was centrifuged at $3000 \mathrm{~g}$ for $10 \mathrm{~min}$. The pellets were collected and washed twice with $50 \mathrm{~mL} 95 \%$ ethanol (v/v). After centrifugation, the pellets were dried at $40{ }^{\circ} \mathrm{C}$ overnight. $150 \mathrm{~mL}$ distilled water was added into the dried flour to extract the water-soluble $\beta$-glucan at $47^{\circ} \mathrm{C}$ for $3 \mathrm{~h}$ using a shaking water bath. Then the suspension was centrifuged and the supernatant was collected and dried as the extracted $\beta$-glucan. The molecular weight of extracted $\beta$-glucan was measured by HPLC according to the literature report using pullulan molecular weight standard (Megazyme International Ireland), and the oat starch was extracted according to our previous work. ${ }^{19,28}$ Then, the samples of oat bran (in a concentration containing $0.5 \%$ starch $\mathrm{w} / \mathrm{v}$ ) and extracted $\beta$-glucan (mixed with oat starch in the same ratio as in oat bran) were cooked in a boiling water bath for 20 min with continuous stirring, and the viscosity of the cooked samples was measured at room temperature $\left(22^{\circ} \mathrm{C}\right)$ along a shear rate from 0.1-100 $\mathrm{s}^{-1}$ using an AR-G2 rheometer (TA Instruments-Waters LLC, Shanghai, China) with a cone plate.

\section{Semi-purified chow diets and mouse study}

Four different chow diets were prepared (Trophic Animal Feed High-Tech Co. Ltd, China) based on our formula (ESI Table $\mathrm{S} 1 \dagger$ ): normal control diet (low fat diet, LF), high fat control diets (HF), HF containing $4 \% \beta$-glucan in a bran form (HFBF), and $\mathrm{HF}$ containing $4 \% \beta$-glucan in an extracted physical from (HFEF).

Three-week-old male C57BL/6J mice $(n=40)$ were purchased from Slack (Suzhou, China), and housed in cages kept on a 12 : 12 light: dark cycle under constant temperature $\left(24^{\circ} \mathrm{C}\right)$ and controlled humidity (55\%). After 1 week of assimilation, the mice were randomly assigned into four groups ( $n=10$ /group) fed on the experimental diets for 6 weeks with free access to food and water. Their body weight was measured once a week and daily food intake was also recorded by weighing the left diets every day, and the daily food intake averaged during each week was presented. The animal study was approved by the Animal Ethics Committee of Jiangnan University (approved no. 2015000511811).

\section{Oral glucose tolerance test (OGTT)}

At the end of 6 weeks feeding on different diets, the oral glucose tolerance test was carried out on the mice after subjecting to an 
overnight fasting. Blood samples were obtained from the tail vein of the mice and blood glucose levels were measured using the One-touch Ultra glucometer and One-touch Ultra test strips (LifeScan, Inc, USA). Then, glucose $\left(2 \mathrm{~g} \mathrm{~kg}^{-1}\right.$ of body weight) was administrated to the mice via the epi-gastric route. Finally, the blood glucose levels were measured every $30 \mathrm{~min}$ for 2 hours $(0$, $30,60,90$ and $120 \mathrm{~min}$ ). AUC (area under the curve of glycemic response) was calculated according to the OGT profile based on the software of Origin 8.5 (OriginLab Corporation USA).

\section{Blood and tissue sampling}

At the end of the study, animals were fasted for $12 \mathrm{~h}$, the mice were then anesthetized with ether, and the blood samples were collected from orbital sinus and dropped into tubes held in an ice bath. After clotting for $2 \mathrm{~h}$ at $4{ }^{\circ} \mathrm{C}$, the tube was centrifuged at $1000 \mathrm{rpm}$ at $4{ }^{\circ} \mathrm{C}$ for $5 \mathrm{~min}$ to collect the serum samples. Then, the mice were sacrificed by cervical dislocation, and the tissues of the liver and small intestine were excised. All the collected serum samples and tissues were stored at $-80{ }^{\circ} \mathrm{C}$ for further analysis.

\section{Serum measurements}

The serum glucose concentration was determined by a diagnostic kit (Nanjing Jiancheng Bioengineering Inst., Nanjing, China) according to the kit instruction. Serum insulin level was measured with an ELISA assay kit (Cusabio Biotech Co., Ltd., Hubei, China) with a microplate reader (SpectraMax M5, Molecular Devices, USA). HOMA-IR and ISI expressed as indices of insulin resistance and insulin sensitivity, respectively, were calculated using the homeostasis model assessment: HOMA-IR $=$ fasting glucose $\left(\mathrm{mmol} \mathrm{L}^{-1}\right) \times$ fasting insulin $\left(\mu \mathrm{U} \mathrm{mL} L^{-1}\right) / 22.5$, ISI $=\ln \left(1 /\right.$ fasting glucose $\left(\mathrm{mmol} \mathrm{L}^{-1}\right) \times$ fasting insulin $\left.\left(\mu \mathrm{U} \mathrm{mL} L^{-1}\right)\right)$. Other serum parameters of glycated hemoglobin of HbA1c, hypothalamic proopiomelanocortin (POMC), and neuropeptide Y (NPY) were determined using corresponding ELISA kits (Shanghai Changjin Biological Co. Ltd, China) according to the instruction manual.

\section{Hepatic glucose metabolism-related enzymes and glycogen determination}

The liver tissue and 9 volumes (w/v) ice-cold buffer $(50 \mathrm{mM}$ sodium HEPES, $100 \mathrm{mM} \mathrm{KCl}, 1 \mathrm{mM}$ EDTA, $5 \mathrm{mM} \mathrm{MgCl}_{2}$, and $10 \mathrm{mM}$ 2-mercaptoethanol, $\mathrm{pH}$ 7.0) were mixed, and the liver tissue was homogenized by an Omni general laboratory homogenizer (Omni International, USA) at a high speed for 30$40 \mathrm{~s}^{-1}$. After centrifugation $(10000 \mathrm{~g})$ for $30 \mathrm{~min}$ at $4{ }^{\circ} \mathrm{C}$, the supernatant as the crude extract was used to measure the enzyme activity. The glucokinase activity was measured using a method of the spectrophotometric continuous assay, and $1 \mu \mathrm{mol}$ glucose-6-phosphate formed per minute per milligram protein was defined as one unit of enzyme activity. ${ }^{29}$ The activity of glucose 6-phosphatase (G6Pase) and liver glycogen content was measured using corresponding ELISA kit (Nanjing Jiancheng Bioengineering Inst., Nanjing, China) according to the instruction manual. BAC Protein Assay was used to measure the protein content.
For small intestine phosphoenolpyruvate carboxykinase (PEPCK) activity measurement, the same procedure as the above was used to prepare the crude extract for each intestine sample, and the PEPCK was measured using an ELISA kit from the same company (Nanjing Jiancheng Bioengineering Inst., Nanjing, China) according to the instruction manual.

\section{Statistical analysis}

The data reported were the average of at least triplicate experimental results, and Statistical Package for the Social Science (SPSS, version 11.5, IBM) was used to analyze the results by oneway analysis of variance (ANOVA) along with Tukey's Post Hoc HSD test. The data were presented as means \pm SEM. The value of $p<0.05$ was considered as statistically significant.

\section{Results and discussion}

\section{The property of prepared oat bran sample and extracted $\beta$ - glucan}

The prepared oat bran sample had a particle size of $349.9 \pm 59.7$ $\mu \mathrm{m}$, and proximate analysis of the sample showed that the $\beta$ glucan was enriched from $6.1 \%$ in the oat flour to a content of $15.6 \%$ along with increased protein content (19.4\%) and decreased content of starch (39.6\%) (Table 1), and the enriched $\beta$-glucan indicates a typical oat bran sample. The extracted $\beta$ glucan (purity $=88 \%$ ) had a molecular weight $\left(M_{\mathrm{w}}\right)$ of $2.05 \times$ $10^{5}$ Da based on HPLC analysis (Fig. 1A), and it is a type of medium viscosity $\beta$-glucan. The intrinsic viscosity was $2.24 \mathrm{dl} \mathrm{g}^{-1}$ based on the Mark-Houwink equation for $\beta$-glucan. ${ }^{30}$ Comparatively, the oat bran sample had a higher viscosity (based on $0.5 \%$ starch, $w / v$ ) than the mixture of oat starch and extracted $\beta$-glucan in the same amount as in the oat bran sample (Fig. 1B) demonstrating the physical form of $\beta$-glucan does have an impact on its rheological properties. The high viscosity of oat bran is likely caused by the interactions among $\beta$ glucan and other components including starch and protein in the bran matrix as reported in literature and our previous study indicating that the bran context (other components and the physical forms) should be taken into consideration to a better understanding of the physiological function of oat $\beta$-glucan. ${ }^{19,31}$

\section{The impact of oat bran on body weight}

As the study was a preventive study, the body weight of the mice (Fig. 2A) was continuously increasing from the initial body weight of $12.61 \pm 0.27 \mathrm{~g}$ up to $26-30 \mathrm{~g}$ at the end of study. However, the body weight of mice on HF diet was significantly higher compared to mice on LF diet starting from week 3 to week 6 demonstrating the effectiveness of high fat diet-induced body weight gain of C57BL $/ 6 \mathrm{~J}$ mice. Actually, an increase of body weight by $27.6 \%$ at week 5 indicates the mice on HF diet has become obese. On the contrast, a significantly lower body weight of mice fed on HFBF was observed at week 4 and week 5 when compared to HF treatment group, and the positive control of mice fed on HFEF also showed a lower body weight at week 5 . Thus, it seems that the anti-obesity effect of $\beta$-glucan is independent of its physical forms, but the HFBF may be more 
Table 1 The composition of the OB- $\beta$-glucan sample compared to the whole oat flour $(\% \mathrm{w} / \mathrm{w})$

\begin{tabular}{lllrrr}
\hline Sample & Starch & Protein & \multicolumn{1}{c}{ Lipids } & \multicolumn{1}{c}{$\beta$-Glucan } & Moisture \\
\hline OB- $\beta$-glucan & $39.6 \pm 0.9$ & $19.4 \pm 0.1$ & $11.4 \pm 0.1$ & $15.6 \pm 0.4$ & $8.3 \pm 0.0$ \\
Whole oat flour & $62.8 \pm 1.6$ & $14.4 \pm 0.2$ & $8.7 \pm 0.2$ & $6.1 \pm 0.3$ & $8.0 \pm 0.5$
\end{tabular}
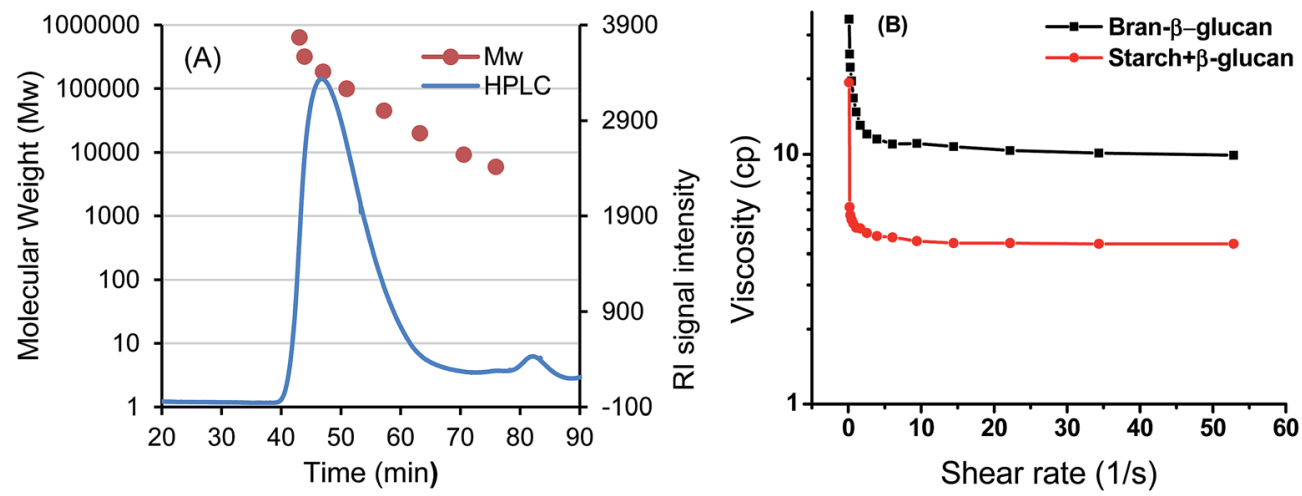

Fig. 1 The molecular weight of extracted $\beta$-glucan (1\%) and the viscosity of prepared OB- $\beta$-glucan sample (containing $0.5 \%$ starch $w / v$ ) compared to a mixture of extracted $\beta$-glucan and starch in the same ratio as in the OB- $\beta$-glucan sample.

efficient to slow the rate of body weight gain with a significant difference from the HF group at an early time of week 4 and a trending low body weight starting from week 2 compared to HFEF treatment.

Analysis of the average daily food intake in each week suggests reduced food intake might be the basis for $\beta$-glucan's anti-obesity function. Certainly, mice fed on either HFBF or HFEF diet had a lower food intake compared to HF group (Fig. 2B) although there was still a significantly higher food intake compared to mice fed on LF diet. Literature studies indicated that reduced satiety by $\beta$-glucan is the main reason of reduced food intake, and gut hormones might play an important role. ${ }^{32}$ Regarding the effect of HFBF and HFEF on the rate of body weight gain, both of them showed similar effect on body weight gain and reduction of food intake, which indicates $\beta$ glucan is the functional component, and the $\beta$-glucan in oat bran, even in a cell wall matrix with a large particle size, was able to exert its health-promoting functions.

\section{Effects of oat bran on the level of fasting blood glucose and HbA1c}

The fasting blood glucose (FBG) is intimately associated with the overall status of glucose metabolism, and impaired fasting glucose (IFG) is characteristic of pre-diabetes. Serum glycated hemoglobin A1c (HbA1c), which is formed by nonenzymatic glycation of hemoglobin after exposure to blood glucose, especially a high level of fasting blood glucose, reflects the status of dysglycemia. The combination of IFG and HbA1c can be used as diagnostic criteria to identify subjects with an increased risk of progression from pre-diabetes to diabetes. ${ }^{33}$ In the current study, when both FBG and HbA1c were measured at the second week, almost no changes were found among all the groups (data not shown). However, at the end of study (week 6), the levels of fasting blood glucose and glycated hemoglobin (HbA1c) were significantly increased in the HF-treated group indicating impaired fasting glucose. When oat bran was incorporated into the HF diet, although the level of FBG did not show significant decrease after six weeks fed on HFBF diet (Fig. 3A), the level of HbA1c was significantly decreased (Fig. 3B) compared to $\mathrm{HF}$ group demonstrating the health potential of $\beta$-glucan in oat bran, just like the extracted counterpart (HFEF), to glucose homeostasis.

\section{Effects of oat bran on oral glucose tolerance (OGTT) and insulin sensitivity}

Insulin resistance is commonly accompanied with obesity that is one main risk factor for type 2 diabetes. ${ }^{24}$ Except for the diagnostic biomarker of FBG and HbA1c for pre-diabetes, glucose tolerance impairment is another measure to reflect the abnormality of glucose homeostasis that is associated with insulin sensitivity. At the end of week 6, the oral glucose tolerance test (OGTT) was carried out, and the results showed a significant difference between LF group and other groups at the initial time of OGTT, which is the FBG (Fig. 4A). However, no significant difference was observed between HF and LF groups in the glucose peak or the area under the curve, which is probably because of the high insulin level in the HF group to suppress the increase of postprandial glucose. ${ }^{34}$ Only the postprandial glucose level of HFBF group decreased significantly at 30 and 60 min when compared to HF group. Consistently, a significant reduction of the area under the curve (AUC) was also observed for HFBF (Fig. 4B). Regarding the effect of HFEF on OGTT profiles, no significant difference was observed when compared to HF and HFBF groups. Thus, although the $\beta$-glucan in oat bran is more efficient to reduce the postprandial glycemia in OGTT test when compared to HF group, no significant 

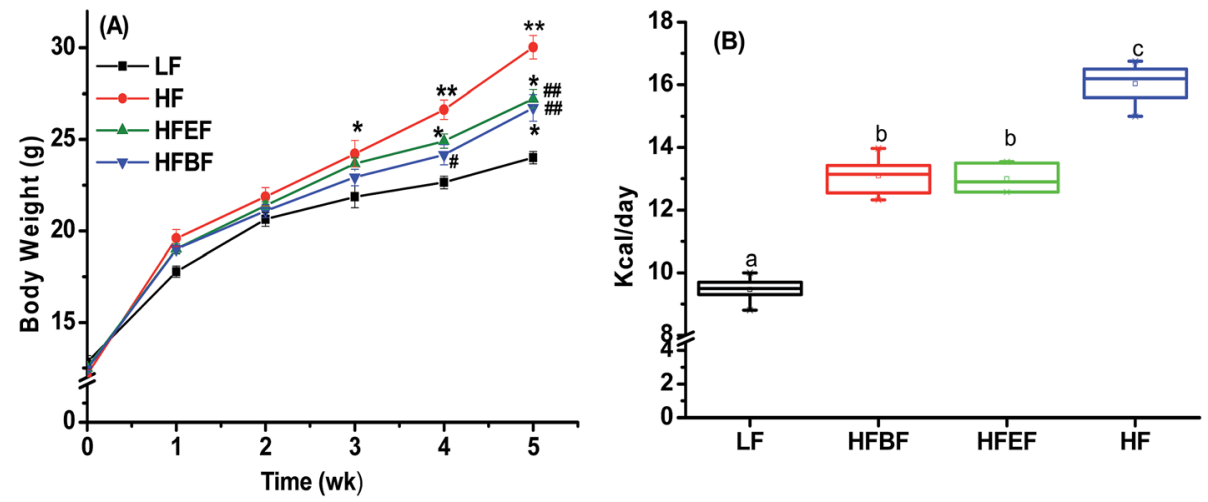

Fig. 2 The changes of body weight (A) and daily food intake (B). LF: low-fat diet, HF: high-fat diet, HFBF: high-fat diet with OB- $\beta$-glucan, HFEF: high-fat diet with extracted $\beta$-glucan. Compared to LF * $(p<0.05), * *(p<0.01)$, compared to HF \#(p<0.05), \#\#(p<0.01). Different letters in (B) represent significant difference at $p<0.05$.
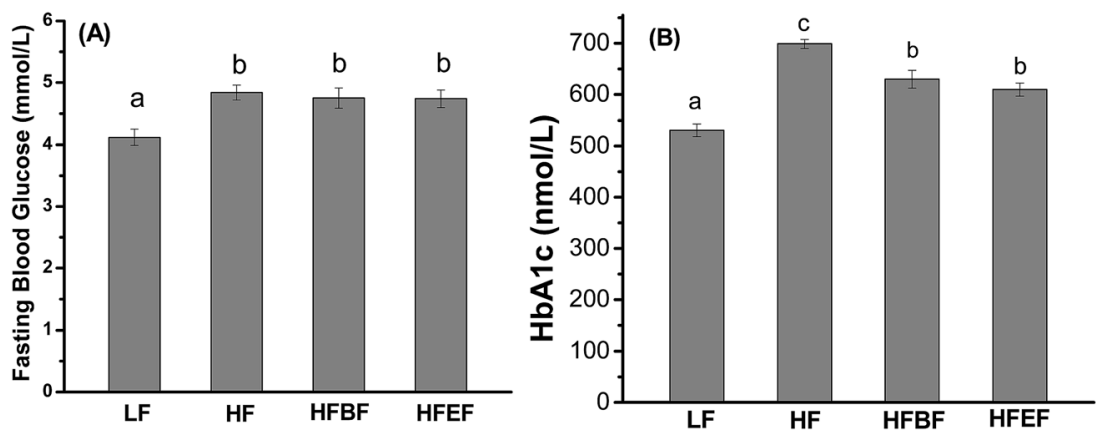

Fig. 3 The fasting blood glucose concentration (A) and the concentration of serum HbA1c (B) after fed on different diets for 6 weeks. Different letters represent statistical significance at $p<0.05$.
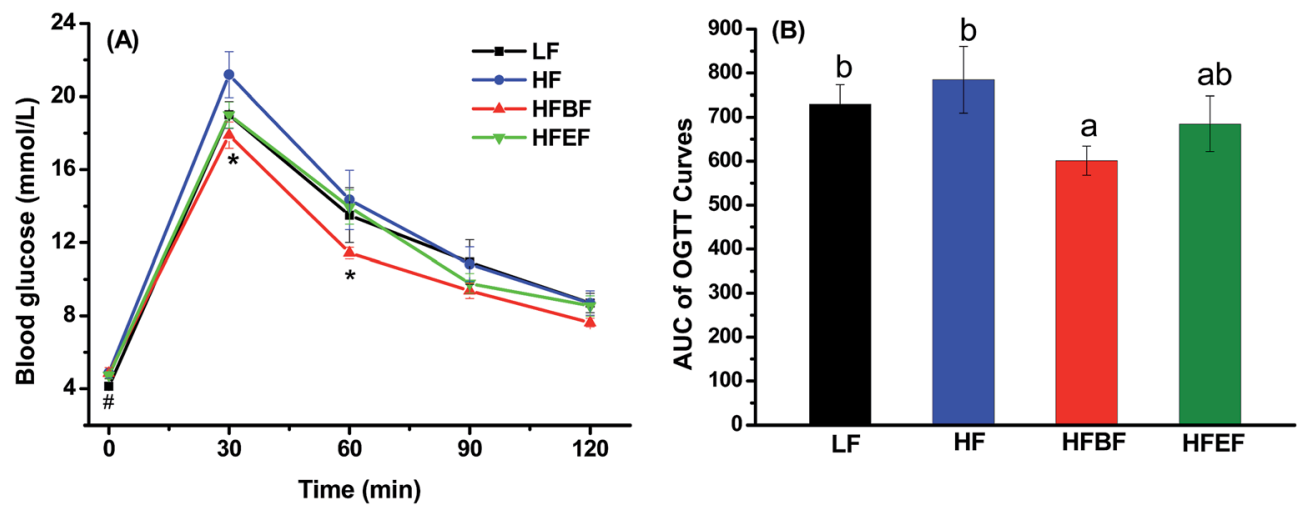

Fig. 4 Postprandial blood glucose profiles (A) and area under the glycemic response curve (AUC) (B) in oral glucose tolerant test of mice fed on different diets. Different letters represent significant difference at $p<0.05$. LF group is significantly different from other groups\#, and HFBF is significantly different from HF groups* at $p<0.05$.

difference was found when it is compared to HFEF group, indicating the $\beta$-glucan in oat bran is also similar to its extracted counterpart concerning the glycemic control.

Insulin resistance is a common feature of obesity and prediabetes with elevated blood glucose level and disordered glucose homeostasis. In the current study, mice fed on either HFBF or HFEF diet have reduced insulin resistance indices approaching to the LF control group (Table 2). Thus, HF diet not only induced an increase in body weight gain but also caused a reduction of insulin sensitivity while the addition of $\beta$-glucan in the HF diet can ameliorate the insulin resistance, which is consistent with literature report on improved insulin sensitivity using extracted $\beta$-glucan as the treatment ingredient. $^{35}$ 
Table 2 The fasting serum insulin in mice fed on different cow diets $(\bar{X}$ $\pm \mathrm{SEM}^{a}$

\begin{tabular}{llll}
\hline Diet group & FINS & ISI & HOMA-IR \\
\hline LF & $1.85 \pm 0.02^{\mathrm{a}}$ & $1.08 \pm 0.02^{\mathrm{a}}$ & $0.34 \pm 0.01^{\mathrm{a}}$ \\
HF & $2.41 \pm 0.07^{\mathrm{b}}$ & $0.65 \pm 0.02^{\mathrm{b}}$ & $0.52 \pm 0.02^{\mathrm{b}}$ \\
HFBF & $1.87 \pm 0.03^{\mathrm{a}}$ & $0.91 \pm 0.01^{\mathrm{a}}$ & $0.40 \pm 0.03^{\mathrm{c}}$ \\
HFEF & $1.89 \pm 0.01^{\mathrm{a}}$ & $0.92 \pm 0.02^{\mathrm{a}}$ & $0.39 \pm 0.01^{\mathrm{c}}$
\end{tabular}

${ }^{a}$ Note: $n=10$, different superscripts (a, b, and c) means significant difference at $p<0.05$.

\section{Effects of $\boldsymbol{\beta}$-glucan on glucose metabolism}

In order to further the understanding of the beneficial impact of OB- $\beta$-glucan on glucose homeostasis, the hepatic glucose metabolism and gluconeogenesis in the small intestine were investigated (Fig. 5). HF diet substantially decreased the glycogen content and activity of glucokinase (GK) in the liver, which indicates the occurrence of glycogenolysis of glycogen and reduced glucose disposal capability of the liver with reduced activity of GK. This result was consistent with increased FBG is HF-fed mice. However, when $4 \% \beta$-glucan was added into the HF diet, the glycogen content increased to the level of the LF control for HFBF group, and even higher than the LF control for HFEF group. The GK activity also returned to the level of the LF control group while no significant changes were found for the activity of G6Pase (convert G-6-P to glucose into blood circulation) even though a lower trending for HFBF and HFEF groups.

Glucokinase is well-known as a hepatic glucose disposer that phosphorylate glucose to G-6-P favoring glycogen synthesis leading to a decrease of blood glucose. Thus, hepatic glucokinase is a critical player to a normal blood glucose control. ${ }^{36,37} \mathrm{In}$ our current study, the GK activity was restored, and the glycogen content also significantly increased when fed on HFBF and HFEF diets. These changes were beneficial to glucose homeostasis and were consistent with the increased insulin sensitivity. However, the fasting blood glucose (FBG) level (Fig. 3) did not show significant improvement even the high GK activity favors the decrease of blood glucose, indicating the complexity of FBG regulation, which needs further study by either extending the treatment time or increasing the dosage of $\beta$-glucan.

\section{Effect of $\beta$-glucan on the changes of NPY and POMC}

In our current study, we observed a significant slow rate of body weight gain in HFBF and HFEF groups compared to HF group after just 5 weeks treatment. A lower body weight is either due to decreased energy intake or increased energy expenditure in
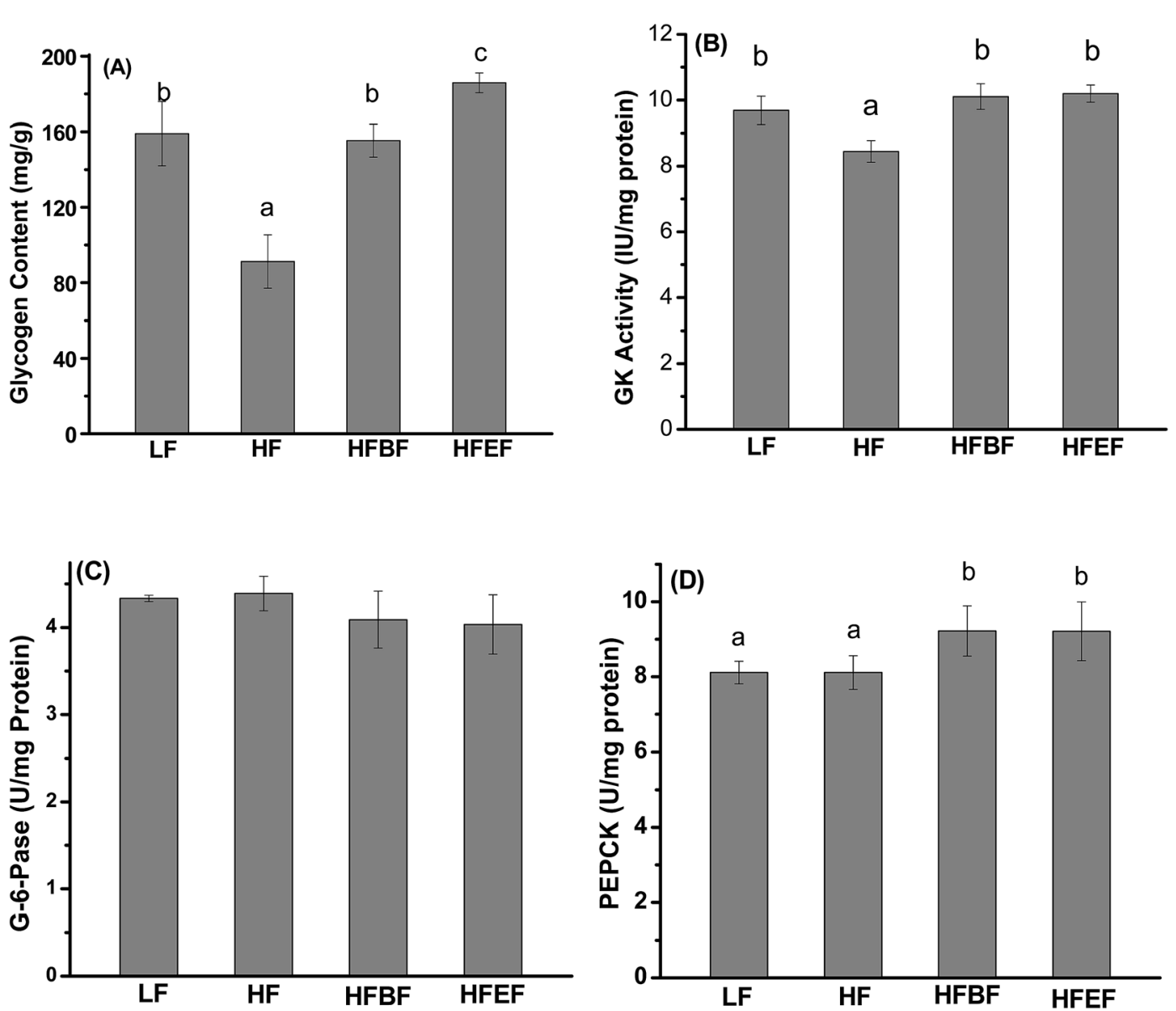

Fig. 5 Glucose metabolism including hepatic glycogen content (A), the activity of glucokinase (B), G6Pase (C) and intestinal PEPCK (D). Different letters represent statistical significance at $p<0.05$. 

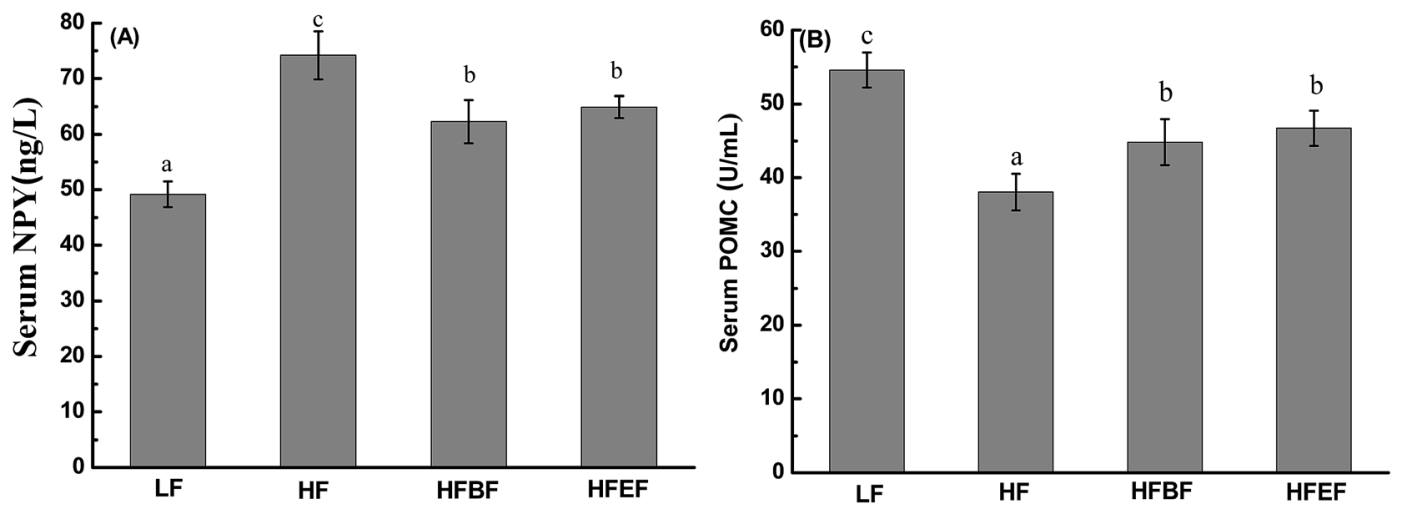

Fig. 6 The impact of $\beta$-glucan on serum neural peptide Y (NPY) (A) and POMC (B). Different letters represent statistical significance at $p<0.05$.

which the central nervous system plays a critical role, particularly the hypothalamus. ${ }^{38}$ The arcuate nucleus within the hypothalamus is key to the regulation of appetite and food intake involving orexigenic (appetite stimulating) neuropeptide Y (NPY) and anorexigenic (appetite suppressing) pro-opiomelanocortin (POMC) neuropeptides. ${ }^{39,40}$ In the current study, we showed a significant increase of NPY and decrease of POMC in mice fed on HF diet (Fig. 6), which is correlated to the increased rate of body weight gain due to a high-calorie intake (Fig. 2). However, when $4 \% \beta$-glucan was added to the HF diet, the serum NPY was significantly decreased while the POMC was increased, which is probably the reason for a slower rate of body weight gain of mice fed on HFBF and HFEF diet with reduced food intake.

According to a previous study, intestinal gluconeogenesis (IGN) was a critical player in the fermentable dietary fiberinduced beneficial effect on glucose and energy homeostasis. ${ }^{51}$ In the current study, the enzyme activity of PEPCK in the small intestine, which is an indicator of gluconeogenesis, was increased significantly in mice fed on $\beta$-glucan-containing $\mathrm{HF}$ diet. The glucose from IGN, as a metabolic signal, can be detected by a hepatoportal glucose sensor of SGLT3 to transmit the signal to the central nervous system to balance energy metabolism. ${ }^{41,42}$

\section{Mechanism of health benefit of OB- $\beta$-glucan}

There have been numerous studies on the health benefits of $\beta$ glucan using either the extracted $\beta$-glucan or bran form $\beta$ glucan (the experimental material is a type of oat bran) that is the most common ingredient in a whole grain food of oat or barley. Whole grain oat food is well known for its health benefits, but the mechanism to these health benefits is still a challenge as the complexity of the food components. In the current investigation using the $\beta$-glucan in oat bran, which is closer to whole grain food ingredient, similar health benefits of improved glucose homeostasis and lower rates of body weight gain were observed for both the extracted $\beta$-glucan and the bran-form $\beta$-glucan, confirming the $\beta$-glucan is the dominant functional component. Although the phytochemicals are generally considered as health-promoting components, their content in oat bran might not be high enough to show observable effects. ${ }^{43}$ On the other hand, the fasting blood glucose, which is an important indicator of glucose homeostasis, did not show significant changes in HFBF and HFEF-fed mice, the reason is likely due to the relatively low dose level of $\beta$-glucan in the oat bran. Certainly, when we used $8 \% \beta$-glucan (extracted form) to treat the mice, significant reduction of FBG was achieved accompanying substantial increase of liver glycogen and the activities of GK and PEPCK (Fig. S1 and Table S2 $\dagger$ ), further demonstrating the function of $\beta$-glucan in glucose homeostasis.

Regarding the mechanism of $\beta$-glucan's influence on glucose homeostasis, there has been a report that gut-brain axis was assumed as the basis for reduced food intake and improved insulin sensitivity. ${ }^{13}$ Reduced pancreatic cell apoptosis and hormone secretion were also considered as the basis for the hypoglycemic effect of oat product. ${ }^{44}$ However, there were not enough details in those studies to conclude the action mechanism. In the current study, simultaneous changes of hepatic glucose metabolism, small intestinal gluconeogenesis, and the neural peptide indicate that there might be an intricate interactions among the gut microbiome (fermentation), liver and the central nervous system, and the health benefit is likely achieved through the activation of the gut-brain-liver axis by $\beta$-glucan. Specifically, $\beta$-glucan is a fermentable dietary fiber, and the fermentation products of short chain fatty acids (SCFA) as reported in literature have been shown to be important to prevent high-fat diet induced obesity through direct hypothalamic neuron activation (acetate) or intestinal gluconeogenesismediated activation of gut-neural circuit (propionate and butyrate). ${ }^{45-51}$ Thus, the activation of the central nervous system through short chain fatty acid generated by $\beta$-glucan fermentation might be the basis of the observed health benefit of improved insulin sensitivity and glucose homeostasis with the concomitantly decreased rate of body weight gain.

Although oat bran and extracted $\beta$-glucan have different physiochemical properties, they did elicit similar long-term health effect on glucose metabolism, which indicates that the oat $\beta$-glucan is the predominant functional component to the induced physiological effects. The $\beta$-glucan in oat bran, even embedded in a cell wall matrix with a particle physical form in the sample, might be utilized completely in the body with an slight better OGTT profiles and seemly more efficient body weight reduction effect, which is likely caused by a possible extended "second meal effect" due to a slow fermentation of $\beta$ - 
glucan in a cell wall matrix form. ${ }^{52}$ Thus, manipulation of the oat bran sample with different particle sizes to simultaneously attenuate acute postprandial glycaemia (first meal effect, might be due to the physical entrapment of starch by $\beta$-glucancontained cell wall matrix) and to maintain its long-term health benefits (through slow fermentation of $\beta$-glucan and possibly other materials) warrants further investigation to maximize the health benefit of $\beta$-glucan in a whole grain context.

\section{Conclusions}

Oat bran, as $\beta$-glucan-enriched ingredient, is often used to produce oat-based functional foods, and $\beta$-glucan is considered as the fundamental functional component for its health benefits. The extracted $\beta$-glucan is also used in functional food production. After long-term feeding study using the C57BL/6J mouse model, high-fat diet treatment significantly increased the body weight, fasting blood glucose, glycated hemoglobin, and insulin resistance when compared to mice fed on low fat diet. In contrast, both oat bran and the extracted $\beta$-glucan in the HF-diet significantly improved the insulin sensitivity and body weight gain. The analysis of gluconeogenesis-related enzymes and liver glucose metabolism as well as the neuro-peptide of proopiomelanocortin and neuropeptide $\mathrm{Y}$ suggest $\beta$-glucan in oat bran is the predominant functional component to the health effects of oat bran on glucose homeostasis. However, the actual mechanism still needs further investigation from the viewpoint of fiber fermentation.

\section{Conflicts of interest}

The authors declare that there are no conflicts of interest.

\section{Abbreviation}

$\begin{array}{ll}\text { HF } & \text { High-fat diet } \\ \text { LF } & \text { Low-fat control diet } \\ \text { HFBF } & \text { High-fat diet containing oat grain form } \beta \text {-glucan }(4 \%) \\ \text { HFEF } & \begin{array}{l}\text { High-fat diet containing extracted oat } \beta \text {-glucan }(4 \%) \\ \text { (positive control of HFBF) }\end{array} \\ \text { OGTT } & \text { Oral glucose tolerance test } \\ \text { NPY } & \text { Neural peptide Y } \\ \text { POMC } & \text { Proopiomelanocortin neural peptide } \\ \text { GK } & \text { Glucokinase } \\ \text { AACC } & \text { Association of American Cereal Chemists } \\ \text { PEPCK } & \text { Phosphoenolpyruvate carboxykinase } \\ \text { HbA1c } & \text { Glycated hemoglobin A1c } \\ \text { FBG } & \text { Fasting blood glucose } \\ \text { FINS } & \text { Fasting insulin } \\ \text { ISI } & \text { Insulin sensitivity index }\end{array}$

\section{Acknowledgements}

The research is supported by the National Natural Science Foundation of China No. 31471585 and the Fundamental Research Funds for the Central Universities (JUSRP51616B).

\section{Notes and references}

1 C. J. Seal and I. A. Brownlee, Proc. Nutr. Soc., 2015, 74, 313319.

2 M. Sadiq Butt, M. Tahir-Nadeem, M. K. Khan, R. Shabir and M. S. Butt, Eur. J. Nutr., 2008, 47, 68-79.

3 M. Alminger and C. Eklund-Jonsson, Eur. J. Nutr., 2008, 47, 294-300.

4 H.-C. Chang, C.-N. Huang, D.-M. Yeh, S.-J. Wang, C.-H. Peng and C.-J. Wang, Plant Foods Hum. Nutr., 2013, 68, 18-23.

5 P. L. B. Hollænder, A. B. Ross and M. Kristensen, Am. J. Clin. Nutr., 2015, 102, 556-572.

6 J. M. Keenan, J. J. Pins, C. Frazel, A. Moran and L. Turnquist, J. Fam. Pract., 2002, 51, 369.

7 D. A. Brockman, X. Chen and D. D. Gallaher, Eur. J. Nutr., 2013, 52, 1743-1753.

8 K. M. Queenan, M. L. Stewart, K. N. Smith, W. Thomas, R. G. Fulcher and J. L. Slavin, Nutr. J., 2007, 6, 6.

9 D. A. Kerckhoffs, G. Hornstra and R. P. Mensink, Am. J. Clin. Nutr., 2003, 78, 221-227.

10 Y. Brummer, R. Duss, T. M. S. Wolever and S. M. Tosh, Cereal Chemistry Journal, 2012, 89, 255-261.

11 J. T. Braaten, P. J. Wood, F. W. Scott, M. S. Wolynetz, M. K. Lowe, P. Bradley-White and M. W. Collins, Eur. J. Clin. Nutr., 1994, 48, 465-474.

12 Q. Wang and P. R. Ellis, Br. J. Nutr., 2014, 112(suppl. 2), S4S13.

13 X. F. Huang, Y. Yu, E. J. Beck, T. South, Y. Li, M. J. Batterham, L. C. Tapsell and J. Chen, Mol. Nutr. Food Res., 2011, 55, 1118-1121.

14 N. Lin, Y. Li, L. Tang, J. Shi and Y. Chen, Mol. Nutr. Food Res., 2013, 57, 1291-1294.

15 Y. Wang, N. P. Ames, H. M. Tun, S. M. Tosh, P. J. Jones and E. Khafipour, Front. Microbiol., 2016, 7, 129.

16 R. A. Burton, M. J. Gidley and G. B. Fincher, Nat. Chem. Biol., 2010, 6, 724-732.

17 S. N. Kiemle, X. Zhang, A. R. Esker, G. Toriz, P. Gatenholm and D. J. Cosgrove, Biomacromolecules, 2014, 15, 1727-1736.

18 AACC-International, 1989, http://www.aaccnet.org/ initiatives/definitions/Documents/OatBran/OatBran.pdf.

19 J. Zhang, K. Luo and G. Zhang, J. Cereal Sci., 2017, 73, 84-90.

20 S. Ibrugger, M. Kristensen, M. W. Poulsen, M. S. Mikkelsen, J. Ejsing, B. M. Jespersen, L. O. Dragsted, S. B. Engelsen and S. Bugel, J. Nutr., 2013, 143, 1579-1585.

21 D. P. Belobrajdic, S. A. Jobling, M. K. Morell, S. Taketa and A. R. Bird, Nutr. Res., 2015, 35, 162-168.

22 H. J. Kim and P. J. White, J. Agric. Food Chem., 2012, 60, 62176222.

23 M. Qatanani and M. A. Lazar, Genes Dev., 2007, 21, 14431455.

24 N. Tapola, H. Karvonen, L. Niskanen, M. Mikola and E. Sarkkinen, Nutr., Metab. Cardiovasc. Dis., 2005, 15, 255-261.

25 H. J. Kim and P. J. White, J. Agric. Food Chem., 2012, 60, 52375242.

26 M. U. Beer, E. Arrigoni and R. Amado, Cereal Chem., 1996, 73, 58-62. 
27 H. J. Kim and P. J. White, J. Agric. Food Chem., 2013, 61, 32703277.

28 R. C. Eerlingen, M. Deceuninck and J. A. Delcour, Cereal Chem., 1993, 70, 345-350.

29 A. L. Davidson and W. J. Arion, Arch. Biochem. Biophys., 1987, 253, 156-167.

30 X. Zheng, L. Li and Q. Wang, Int. J. Mol. Sci., 2011, 12, 15631574.

31 Y. Liu, T. B. Bailey and P. J. White, J. Agric. Food Chem., 2010, 58, 9198-9203.

32 E. J. Beck, S. M. Tosh, M. J. Batterham, L. C. Tapsell and X.-F. Huang, Mol. Nutr. Food Res., 2009, 53, 1343-1351.

33 Y. Heianza, S. Hara, Y. Arase, K. Saito, K. Fujiwara, H. Tsuji, S. Kodama, S. D. Hsieh, Y. Mori, H. Shimano, N. Yamada, K. Kosaka and H. Sone, Lancet, 2011, 378, 147-155.

34 Y. Lee, E. D. Berglund, X. Yu, M. Y. Wang, M. R. Evans, P. E. Scherer, W. L. Holland, M. J. Charron, M. G. Roth and R. H. Unger, Proc. Natl. Acad. Sci. U. S. A., 2014, 111, 13217-13222.

35 J. Zheng, N. Shen, S. Wang and G. Zhao, Food Nutr. Res., 2013, 57, DOI: 10.3402/fnr.v57i0.22754.

36 L. Agius, Biochem. J., 2008, 414, 1-18.

37 L. Agius, Diabetes, 2009, 58, 18-20.

38 S. C. Heinrichs, F. Menzaghi, E. M. Pich, R. L. Hauger and G. F. Koob, Brain Res., 1993, 611, 18-24.

39 A. C. Könner, T. Klöckener and J. C. Brüning, Physiol. Behav., 2009, 97, 632-638.

40 R. D. Cone, M. A. Cowley, A. A. Butler, W. Fan, D. L. Marks and M. J. Low, Int. J. Obes., 2001, 25(suppl. 5), S63-S67.

41 F. Delaere, A. Duchampt, L. Mounien, P. Seyer, C. Duraffourd, C. Zitoun, B. Thorens and G. Mithieux, Mol. Metab., 2013, 2, 47-53.
42 G. Mithieux, Neuroendocrinology, 2014, 100, 89-94.

43 S. Sangwan, R. Singh and S. K. Tomar, J. Innova. Bio, 2014, 1, 3-14.

44 R. L. Shen, F. L. Cai, J. L. Dong and X. Z. Hu, J. Agric. Food Chem., 2011, 59, 8895-8900.

45 N. Beraza and C. Trautwein, Hepatology, 2008, 48, 10111013.

46 P. J. Wood, E. Arrigoni, S. S. Miller and R. Amado, Cereal Chem., 2002, 79, 445.

47 A. Kaur, D. J. Rose, P. Rumpagaporn, J. A. Patterson and B. R. Hamaker, J. Food Sci., 2011, 76, H137-H142.

48 G. den Besten, A. Bleeker, A. Gerding, K. van Eunen, R. Havinga, T. H. van Dijk, M. H. Oosterveer, J. W. Jonker, A. K. Groen, D. J. Reijngoud and B. M. Bakker, Diabetes, 2015, 64, 2398-2408.

49 I. Kimura, K. Ozawa, D. Inoue, T. Imamura, K. Kimura, T. Maeda, K. Terasawa, D. Kashihara, K. Hirano, T. Tani, T. Takahashi, S. Miyauchi, G. Shioi, H. Inoue and G. Tsujimoto, Nat. Commun., 2013, 4, 1829.

50 G. Frost, M. L. Sleeth, M. Sahuri-Arisoylu, B. Lizarbe, S. Cerdan, L. Brody, J. Anastasovska, S. Ghourab, M. Hankir, S. Zhang, D. Carling, J. R. Swann, G. Gibson, A. Viardot, D. Morrison, E. Louise Thomas and J. D. Bell, Nat. Commun., 2014, 5, 3611.

51 F. De Vadder, P. Kovatcheva-Datchary, D. Goncalves, J. Vinera, C. Zitoun, A. Duchampt, F. Backhed and G. Mithieux, Cell, 2014, 156, 84-96.

52 F. Brighenti, L. Benini, D. Del Rio, C. Casiraghi, N. Pellegrini, F. Scazzina, D. J. Jenkins and I. Vantini, Am. J. Clin. Nutr., 2006, 83, 817-822. 